\title{
Crustal anisotropy of Taihang Mountain Range using azimuthal variation of receiver functions*
}

\author{
TIAN Bao-feng^ (田宝峰) LI Juan (李娟) YAO Zhen-xing (姚振兴) \\ Institute of Geology and Geophysics, Chinese Academy of Sciences, Beijing 100029, China
}

\begin{abstract}
We discussed the possibility of studying crust anisotropy by analyzing azimuthal variation of the receiver functions and presented a technique for computing the transmission response of a flat-layered medium with arbitrarily oriented hexagonally symmetric anisotropy using the reflectivity algorithm. Using this method we investigated the crust anisotropy of Taihang Mountain Range (TMR). Our result shows that there is significant anisotropy with a slow symmetry axis in the upper crust and a fast symmetry axis in the lower crust. The anisotropy magnitude of about $8 \% \sim 15 \%$ is found in the upper crust and a smaller magnitude of about $3 \% \sim 5 \%$ in the lower crust. Orientation of the symmetry axes and the depth of anisotropy appearance as deduced from the seismic records of four individual seismic stations are different from each other. The crust anisotropy beneath the four stations may be associated with the local crustal fabrics in a small area.
\end{abstract}

Key words: receiver function; anisotropy; azimuthal pattern; Taihang Mountain Range CLC number: P313.2 Document code: A

\section{Introduction}

Seismic anisotropy is the property of the dependence of seismic velocity upon angle. The history of studying on seismic anisotropy is only about half a century, but various scholars have achieved many constructive results. Anderson (1961) and some others studied the principle of seismic wave propagation systematically since 1950s. Hess (1964) discovered the phenomenon of azimuthal anisotropy at Pacific mid-ocean ridge, and provided important geophysical evidences for ocean-floor spreading hypothesis in 1960s. Since Crampin and Booth (1985) discovered the phenomenon of shear wave splitting in 1980s, the technique of shear-wave splitting has become a main and effective method in studying the crust and mantle anisotropy. Anatomizing anisotropy information contained in the seismic waves can provide useful information about the Earth's interior structure, deformation (Tommasi et al, 1999), dynamic processing (Montagner, 1998) and mantle convection (Karato, 1998). Therefore, seismic anisotropy plays an increasingly important role during earth science research, such as theoretical seismology, exploring seismology, geodynamics and earthquake catastrophology.

The evolution of the North China Craton (NCC) is a hot issue of present researches. The different ideas about the history of NCC, such as lithosphere thinning, require urgently other more

\footnotetext{
* Received 2008-03-14; accepted in revised form 2008-05-20.

Foundation item: National Natural Science Foundation of China (40774042 and 90714012).

* Author for correspondence: bftian@mail.iggcas.ac.cn
} 
results from geochemistry and geophysics for verification. Seismic anisotropy gives a new way to get knowledge of the interior structure and status of stress within the plate. Furthermore, we can have a good understanding of mantle convection and plate motion. The most common method at present to study seismic anisotropy is the shear-wave splitting technique (ZHENG and GAO, 1994; LIU et al, 2001; LUO et al, 2004; WU et al, 2007; Iidaka and Niu, 2001; Zhao and Zheng, 2005). The shear-wave splitting technique is developed from a simple principle, but the results obtained by various scholars in the same region or obtained by one scholar with different earthquake records using this technique sometimes are dramatically contradictory to each other. Liu et al (2008) gave a more comprehensive overview of the shear-wave splitting results. They thought that the inconsistency of the previous studies was mostly due to the limited amount of events and different shear-wave splitting measurements. They also gave a reliable research on the seismic anisotropy of North China using much more events records that satisfy one standard defined by them. Though errors can be reduced by improvement of the processing technique, the shear-wave splitting technique could still not give any resolution of the depth distribution of anisotropy. In addition, it can only be applied to the area with horizontal oriented hexagonally symmetric anisotropy (i.e., transverse isotropy with a horizontal axis). Thus a new method is needed to give more details of anisotropy.

Because of its sensitivity to velocity discontinuities in the subsurface, receiver function technique has become a routine procedure used to investigate interior structure of the Earth (LIU et al, 1997; Wu et al, 2007a, b; Owens et al, 1984). Information about Earth's interior anisotropic structure can be remained on the receiver functions with a pattern that varies for different back azimuths on both radial and transverse components. However, the traditional methods, such as migration imaging and waveform inversion techniques that are based on isotropic media assumption, cannot effectively extract these information. Only by computing the response based on anisotropic media assumption, e.g., a model including anisotropy with a symmetric axis of arbitrary orientation, can we obtain the detailed anisotropy information presented on the radial and transverse receiver functions. In recent years, plentiful achievements about crust anisotropy using receiver functions were made by scholars in China and aboard. For example, Levin and Park (1997) studied the crustal anisotropy in the Ural Mountains foredeep from teleseismic receiver functions and the result showed that there are significant anisotropy in a low velocity layer at surface $(\sim 1 \mathrm{~km})$ and in the lower crust (33 40 km); Savage (1998) studied the lower crustal anisotropy and dipping boundaries' effects on receiver functions in New Zealand; Ozacar and Zand (2004) researched the crustal seismic anisotropy at BNS station in central Tibet considering both anisotropy and dipping interface, and showed the implications for deformational style and flow in the crust; waveform modeling using the neighborhood algorithm (NA) applied to receiver functions computed from 11 PASSCAL stations spanning the eastern plateau of Tibet yielded a suit of crustal models that include anisotropy by Sherrington et al (2004), and those models suggested that the Tibetan crust contains $4 \% \sim 14 \%$ anisotropy at different depths and the alignment of the symmetry axis of anisotropy in the surface layer shows a well relationship with crustal fabrics associated with E-W trending faults and sutures.

The method of studying crustal anisotropy using the azimuthal variations of receiver functions can be applied to regions with complex anisotropic feature, from which we can constrain the lateral and depth distribution of anisotropy, as well as other important parameters. In this paper, we first introduced the process of computing the response in anisotropic medium with some important equations, then applied this method to the crustal anisotropy of TMR in North China, and finally gave the seismic evidence of anisotropy existing within the crust. 


\section{Reverberations in anisotropic medium}

\subsection{Transverse isotropy}

The symmetry of elastic media plays an important role in studying crystal anisotropy. In crystallography, the anisotropic medium can be categorized into many kinds of symmetry systems according to its symmetry plane and axis. Different anisotropic systems have different amount of elastic coefficients. The higher the symmetry system, the fewer the elastic coefficients are required to describe the anisotropy. Twenty one elastic coefficients are required for the lowest symmetry system (triclinic systems) comparing with only two elastic coefficients for the highest (isotropic system) (Crampin and Booth, 1985). The category of seismic anisotropy is extended from that of crystal anisotropy. The representative systems usually mentioned in seismology are triclinic, monoclinic, orthorhombic, hexagonal (i.e., transverse isotropic or TI) and isotropic systems.

The TI system is simple but effective to describe the crustal anisotropic medium. It can be subdivided into the following cases: transverse isotropy with a vertical symmetry axis (VTI), transverse isotropy with a horizontal symmetry axis (HTI) and transverse isotropy with an arbitrary orientated symmetry axis. Most of researches (Tsvankin and Thomsen, 1994; ZHENG and ZHANG, 2005; DU and YANG, 2004) usually focus on the VTI and HTI system. However, using the VTI or HTI system to describe the anisotropy in the region with complex structures, such as the orientation of microcracks or strata is not valid to be vertical or horizontal, may introduce significant errors.

The elastic tensor is much more complex to describe the transverse isotropic system with an arbitrary orientated symmetry axis and it is much more difficult to know the meaning as well as the value of each element. By introducing five parameters $(A, B, C, D, E)$ describing the magnitude of anisotropy and two parameters describing the orientation of symmetry axis (azimuth angle $\varphi$ and dip angle $\theta$ ) (Park, 1996), we can get the values of the tensor. For TI system with a symmetry axis oriented $\hat{\boldsymbol{w}}$, the elastic tensor $(\boldsymbol{\Lambda})$ can be expressed as

$$
\boldsymbol{\Lambda}=A \boldsymbol{\Lambda}_{A}+B \boldsymbol{\Lambda}_{B}+C \boldsymbol{\Lambda}_{C}+D \boldsymbol{\Lambda}_{D}+E \boldsymbol{\Lambda}_{E}
$$

where

$$
\left\{\begin{array}{l}
\boldsymbol{\Lambda}_{A}=\boldsymbol{I} \otimes \boldsymbol{I} \\
\boldsymbol{\Lambda}_{B}=\boldsymbol{W} \otimes \boldsymbol{I}+\boldsymbol{I} \otimes \boldsymbol{W} \\
\boldsymbol{\Lambda}_{C}=8 \boldsymbol{W} \otimes \boldsymbol{W}-\boldsymbol{I} \otimes \boldsymbol{I} \\
\boldsymbol{\Lambda}_{D}=(13) \boldsymbol{I} \otimes \boldsymbol{I}+(14) \boldsymbol{I} \otimes \boldsymbol{I}-2 \boldsymbol{I} \otimes \boldsymbol{I} \\
\boldsymbol{\Lambda}_{E}=2\left[(13) \boldsymbol{\Lambda}_{B}+(14) \boldsymbol{\Lambda}_{B}-2 \boldsymbol{\Lambda}_{B}\right]+\boldsymbol{\Lambda}_{D}
\end{array}\right.
$$

where " $\otimes$ " is the tensor product operation named Kronecker product, and $\boldsymbol{W}=\hat{\boldsymbol{w}} \otimes \hat{\boldsymbol{w}}-\boldsymbol{I} / 2$ with $\boldsymbol{I}$ being the identity tensor. The permutation $(i j)$ indicates the interchange between the $i$ th and $j$ th tensor indices, for example, $\{(13) \boldsymbol{I} \otimes \boldsymbol{I}\}_{i j k d}=(13) \delta_{i j} \delta_{k l}=\delta_{k j} \delta_{i l}$.

Some special cases of anisotropic system would suggest knowledge of $A, B, C, D$ and $E$. In isotropic medium, $B=C=E=0, A=\lambda+2 \mu$ and $D=\mu$, where $\lambda, \mu$ are the Lamé parameters. If the axis of symmetry is horizontal, we can express the azimuthal dependence of the quasi-P, quasi-SV and quasi-SH velocities for horizontal propagation in terms of angle $\xi$ from $\hat{\boldsymbol{w}}$ as 


$$
\left\{\begin{array}{l}
\rho \alpha^{2}(\xi)=A+B \cos 2 \xi+C \cos 4 \xi \\
\rho \beta_{\mathrm{SV}}^{2}(\xi)=D+E \cos 2 \xi \\
\rho \beta_{\mathrm{SH}}^{2}(\xi)=D+C(1-\cos 4 \xi)+E
\end{array}\right.
$$

Therefore, $A$ and $D$ denote the average velocity of $\mathrm{P}$ and $\mathrm{S}$ wave, respectively; $B$ and $E$ denote the anisotropy strength; $C$ is the correction term for the elliptical anisotropy (generally $C<<B$ ).

According to the shapes of phase-velocity surfaces, TI medium can be also divided into the one with fast axis and the other with slow axis. If $B>0$ and $E>0$, the velocity of seismic wave propagating along the symmetry axis is fastest, leading to phase-velocity surface that resembles a watermelon, and thus the symmetry is defined "fast" axis. In contrast, if $B<0$ and $E<0$, the phase-velocity surface that resembles a pumpkin with the symmetry axis called "slow" axis, as shown in Figure 1.

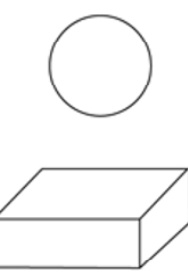

(a)
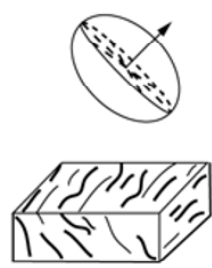

(b)

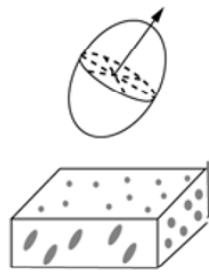

(c)

Figure 1 Illustration of phase velocity surface for different mediums and possible geologic explation

(a) Isotropy, $B=0, E=0$; (b) Anisotropy with a slow symmetry axis, $B<0, E<0$; (c) Anisotropy with a fast symmetry axis, $B>0, E>0 ; B$ and $E$ are parameters of anisotropy magnitude, and the arrowhead shows the orientation of anisotropy symmetry axis

\subsection{Reverberations in TI medium}

With the elastic tensor of an anisotropic layer, three couples of downgoing and upgoing plane wave solutions can be obtained by solving the elastic wave equation, i.e., the vertical slowness (wavenumber) and polarization direction of these plane waves. The general solution of plane wave, $\boldsymbol{u}=U_{0} \exp (i k r-i \omega t)$, satisfies

$$
\left(k^{2} \boldsymbol{C}-\rho \omega^{2}\right) \cdot \boldsymbol{u}=0
$$

where $U_{0}$ is a constant, $\boldsymbol{r}$ is the displacement vector, $\omega$ is the circular frequency, $\boldsymbol{C}=\hat{\boldsymbol{k}} \cdot \boldsymbol{\Lambda} \cdot \hat{\boldsymbol{k}}$ is the Christoffel matrix, $\boldsymbol{k}$ is the wavenumber vector of plane wave, $k=|\boldsymbol{k}|$, and $\hat{\boldsymbol{k}}=\boldsymbol{k} / \boldsymbol{k}$ is the unit wavenumber vector. The three downgoing and upgoing waves in the VTI media share the same horizontal wavenumber (slowness). Setting $\boldsymbol{k}=p \hat{\boldsymbol{x}}+v \hat{\boldsymbol{z}}$ and substituting equation (1) into equation (4) gives

$$
\left(p^{2} \boldsymbol{T}-\rho \boldsymbol{I}+v p \boldsymbol{S}+v^{2} \boldsymbol{R}\right) \cdot \boldsymbol{u}=0
$$

where

$$
\left\{\begin{aligned}
\boldsymbol{T}= & (A-B+C-D+E) \hat{\boldsymbol{x}} \otimes \hat{\boldsymbol{x}}+\left[D+\left(2 w_{x}^{2}-1\right) E\right] \boldsymbol{I}+ \\
& \left(8 w_{x}^{2} C+2 E\right) \hat{\boldsymbol{w}} \otimes \hat{\boldsymbol{w}}+(B-4 C-2 E) w_{x}(\hat{\boldsymbol{w}} \otimes \hat{\boldsymbol{x}}+\hat{\boldsymbol{x}} \otimes \hat{\boldsymbol{w}})+ \\
\boldsymbol{S}= & (A-B+C-D+E)(\hat{\boldsymbol{x}} \otimes \hat{\boldsymbol{z}}+\hat{\boldsymbol{z}} \otimes \hat{\boldsymbol{x}})+4 w_{x} w_{z} E \boldsymbol{I}+16 w_{x} w_{z} C \hat{\boldsymbol{w}} \otimes \hat{\boldsymbol{w}} \\
& (B-4 C-2 E)\left[w_{z}(\hat{\boldsymbol{w}} \otimes \hat{\boldsymbol{x}}+\hat{\boldsymbol{x}} \otimes \hat{\boldsymbol{w}})+w_{x}(\hat{\boldsymbol{w}} \otimes \hat{\boldsymbol{z}}+\hat{\boldsymbol{z}} \otimes \hat{\boldsymbol{w}})\right. \\
\boldsymbol{R}= & (A-B+C-D+E) \hat{\boldsymbol{z}} \otimes \hat{\boldsymbol{z}}+\left[D+\left(2 w_{z}^{2}-1\right) E\right] \boldsymbol{I}+ \\
& \left(8 w_{z}^{2} C+2 E\right) \hat{\boldsymbol{w}} \otimes \hat{\boldsymbol{w}}+(B-4 C-2 E) w_{z}(\hat{\boldsymbol{w}} \otimes \hat{\boldsymbol{z}}+\hat{\boldsymbol{z}} \otimes \hat{\boldsymbol{w}})
\end{aligned}\right.
$$

The Christoffel equation, expressed as $\operatorname{det}\left(p^{2} \boldsymbol{T}-\rho \boldsymbol{I}+v p \boldsymbol{S}+v^{2} \boldsymbol{R}\right)=0$, corresponding to equation (5), 
has sixth order on $v$, which is difficult to be solved. Park (1996) transformed equation (5) from solving the sixth-order roots in $3 \times 3$ system to determine the eigenvalues and eigenvectors in a new $6 \times 6$ system, as is shown in equation $(7)$

$$
\left(\left[\begin{array}{cc}
-\widetilde{\boldsymbol{S}} & -\widetilde{\boldsymbol{T}} \\
\boldsymbol{I} & 0
\end{array}\right]-v\left[\begin{array}{cc}
\boldsymbol{I} & 0 \\
0 & \boldsymbol{I}
\end{array}\right]\right) \cdot\left[\begin{array}{c}
v \boldsymbol{u} \\
\boldsymbol{u}
\end{array}\right]=0
$$

with $\widetilde{\boldsymbol{T}}=R^{-1}\left(p^{2} \boldsymbol{T}-\rho \boldsymbol{I}\right), \widetilde{\boldsymbol{S}}=p \boldsymbol{R}^{-1} \cdot \boldsymbol{S}$ and $\boldsymbol{Q}=\left[\begin{array}{cc}-\widetilde{\boldsymbol{S}} & -\widetilde{\boldsymbol{T}} \\ \boldsymbol{I} & 0\end{array}\right]$. The six eigenvalues of matrix $\boldsymbol{Q}$ are corresponding to the six slowness values $\left(v_{1}, v_{2}, v_{3}, v_{4}, v_{5}, v_{6}\right)$ of the plane waves, which generate the six polarization direction of the plane waves.

In the layered homogenous TI media, the displacement-stress vector in the $k$-th layer is described in terms of the linear combination of the eigenvalues and eigenvectors as follows

$$
\boldsymbol{f}^{(k)}(z)=\boldsymbol{E}^{(k)} \cdot \boldsymbol{\gamma}^{(k)} \cdot \boldsymbol{c}^{(k)}
$$

where $\boldsymbol{E}^{(k)}$ is a $6 \times 6$ matrix, with each row vector denotes its polarization direction and corresponding displacement-stress vector. The first three rows denote the downgoing waves, and the rest denote the upgoing. $\gamma^{(k)}$ is a diagonal matrix composed of six eigenvalues which reads

$$
\gamma^{(k)}(z)=\operatorname{diag}\left\{\mathrm{e}^{i v_{1}\left(z-z_{k-1}\right)}, \mathrm{e}^{i v_{2}\left(z-z_{k-1}\right)}, \mathrm{e}^{i v_{3}\left(z-z_{k-1}\right)}, \mathrm{e}^{i v_{4}\left(z-z_{k}\right)}, \mathrm{e}^{i v_{5}\left(z-z_{k}\right)}, \mathrm{e}^{i v_{6}\left(z-z_{k}\right)}\right\}
$$

where $\boldsymbol{c}^{(k)}$ are the amplitude coefficients of the six plane wave, $\boldsymbol{c}^{(k)}=\left(\boldsymbol{c}_{\mathrm{d}}^{(k)}, \boldsymbol{c}_{\mathrm{u}}^{(k)}\right)^{\mathrm{T}}$, 'd' denotes downgoing and ' $u$ ' denotes upgoing. According to the reflectivity method (Chen, 1993), we obtain

$$
\left\{\begin{array}{l}
\boldsymbol{c}_{\mathrm{u}}^{(1)}=\hat{\boldsymbol{T}}_{\mathrm{u}}^{(1)} \cdot \hat{\boldsymbol{T}}_{\mathrm{u}}^{(2)} \cdots \cdot \hat{\boldsymbol{T}}_{\mathrm{u}}^{(k)} \cdot \boldsymbol{c}_{\mathrm{u}}^{(k+1)} \\
\boldsymbol{c}_{\mathrm{d}}^{(1)}=\hat{\boldsymbol{R}}_{\mathrm{ud}}^{(0)} \cdot \boldsymbol{c}_{\mathrm{u}}^{(1)}
\end{array}\right.
$$

where $\hat{\boldsymbol{T}}_{\mathrm{u}}^{(k)}$ and $\hat{\boldsymbol{R}}_{\mathrm{ud}}^{(k)}$ are the generalized transmission/reflection coefficients at the $k$-th interface, $k=0$ denotes the free surface. Only upgoing incident waves exist in half-space; thus when the incident wave is $\mathrm{P}$ wave, $\boldsymbol{c}_{\mathrm{u}}^{(k+1)}$ can be represented by normalized vector as $(1,0,0)$.

Substituting $\boldsymbol{E}^{(1)}, \boldsymbol{\gamma}^{(1)}$ and $\boldsymbol{c}^{(1)}$ into equation (8) gives the ground surface displacement. The displacement is actually the displacement spectrum as the calculation is implemented in frequency-space domain in fact. The ground surface displacement in time-space domain, i.e., the response of the media, is obtained by an inverse Fourier Transform as shown in Figure 2.

\section{Crustal anisotropy in North China}

\subsection{Data and processing}

A great number of broadband mobile stations with high sensitivity have been deployed in NCC area in recent years, and thus large numbers of digital records offer an excellent opportunity to study the crustal anisotropy using receiver functions. Earthquake seismograms in this paper were chosen from data collected as part of the Northern China Interior Structure Project-II (NCISP-II) of broadband mobile stations. The records with good azimuth coverage collected from the 182 station $\left(115.2^{\circ} \mathrm{E}, 39.1^{\circ} \mathrm{N}\right)$, the 184 station $\left(115.1^{\circ} \mathrm{E}, 39.2^{\circ} \mathrm{N}\right)$, the 189 station 


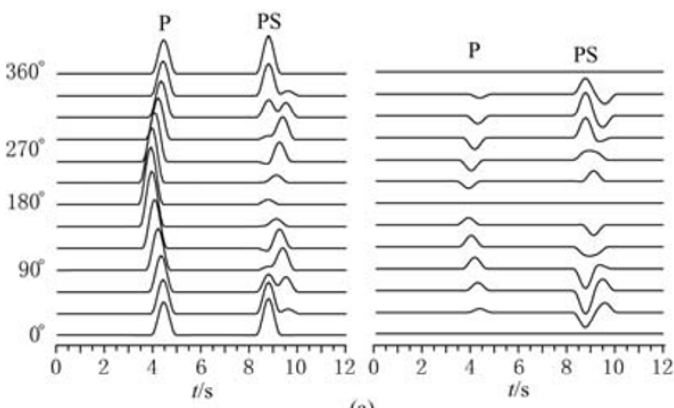

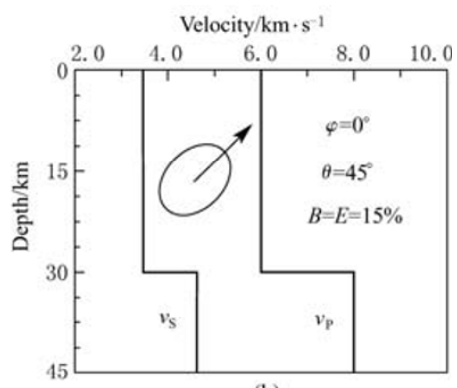

(b)

Figure 2 Reverberations in a flat-layered anisotropic medium (a). Left plot shows radial component and the right shows transverse component; the number at the start of a horizontal trace denotes corresponding back azimuth. $\mathrm{P}$ and PS show the direct $\mathrm{P}$ arrival and $\mathrm{P}$ to $\mathrm{S}$ conversion, respectively. Velocity model used in producing Figure $2 \mathrm{a}(\mathrm{b}) . \varphi$ and $\theta$ are the trend and plunge of the symmetry axis, respectively, and $B$ and $E$ show the anisotropy magnitude

$\left(114.6^{\circ} \mathrm{E}, 39.5^{\circ} \mathrm{N}\right)$ and the 194 station $\left(114.1^{\circ} \mathrm{E}\right.$, $39.7^{\circ} \mathrm{N}$ ) were used in this study. The location of stations and the geologic background of the study region are shown in Figure 3. The four selected mobile stations distribute across the TMR, part of the Central Transform Zone, along the NW-SE direction. The Mesozoic depression basin (i.e., Erdos) and the Cenozoic rift basin are at the west and east of the study area, respectively.

According to the global earthquake catalog, we selected the records of seismic events with magnitude of $M_{\mathrm{b}} \geq 5.0$ from September, 2001 to February, 2003. The records with clear phases and initial arrivals were chosen to compute the receiver functions. Some seismic event records with epicentral distance slightly higher than $90^{\circ}$ or lower than $30^{\circ}$ were also chosen because of the rare amount of events in some azimuths.

The radial and transverse components of

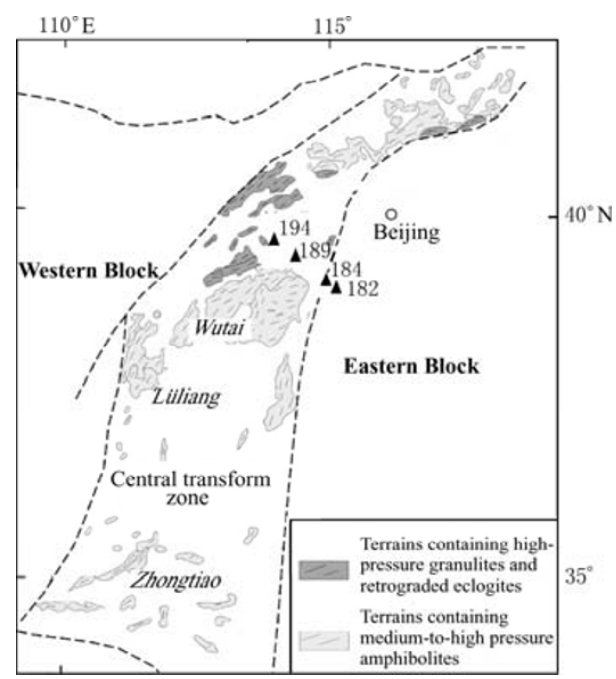

Figure 3 Geologic scheme of the study region and location of the four seismic stations (modified after Lu et al, 2008, Figure 3) receiver functions were computed using the maximum entropy deconvolution method (WU et al, $2003)$ and a Gaussian filter value of $2.5(\alpha=2.5)$ was used. In order to enhance the signal-to-noise ratio and make the azimuthal variation of receiver functions clear, we divided the back azimuth into 36 units by a $10^{\circ}$ interval, and stacked the receiver functions at the same back azimuth range. Thus we got the patterns of receiver functions with an azimuthal variation at each station.

\subsection{Anisotropy and azimuthal variation of receiver functions}

A receiver function waveform is composed of an initial arrival with P polarity, the remainder of the waveforms consisted of $\mathrm{S}$ waves that converted from $\mathrm{P}$ waves at velocity contrasts in the subsurface and their multiple waves. In the absence of anisotropy or lateral heterogeneity, tele- 
seismic converted S waves should remain in the source-receiver plane and have exclusively SV particle motion. Energy would then only be present on the radial component and there would be no waveform variation on the transverse component. The presence of lateral heterogeneity, such as dipping layers, or anisotropy will result in rotation of energy out of the source-receiver plane and conversions from P to both SV and SH particle motion. Except of some differences in details, anisotropy and dipping layers yield similar influence on the receiver functions. When the dipping angle is small, anisotropy and dipping layers both yield patterns of receiver function amplitudes and arrival times that vary for different back azimuths with a symmetric or antisymmetric relation along $180^{\circ}$ ( $\mathrm{Li}$ et al, 2007). The waveform of receiver functions corresponding to the converted $\mathrm{S}$ waves exists double peaks because of shear wave splitting when medium presents anisotropy (TIAN et al, 2008), but no such phenomena for dipping layers. Distinguishing between dipping layers and anisotropy as explanations for systematic back azimuth variation of receiver function appearance is somewhat non-unique, although the differences between them in synthetic or theory. For simplicity, this study deals only with modeling of anisotropy in receiver functions.

\subsection{Example of crustal anisotropy inversion : 189 station}

Because of the numerous anisotropy parameters with tradeoff between each other during waveform inversion, the results will have a fair chance of unique. During the processing, to reduce the non-uniqueness of inversion, we give a primary model obtained by analyzing the arrival time and azimuthal variation of the main phases at first. In addition, some approximation and assumptions have been set before inversion, for example, we assume that the $\mathrm{S}$ wave anisotropy magnitude equals to the $\mathrm{P}$ wave; the ratio of $v_{\mathrm{P}} / v_{\mathrm{S}}$ is 2.0 in surface layer, and 1.73 for lower layers; the density of different layers can be calculated by the formula $\left(\rho=0.32 v_{\mathrm{P}}+0.77\right)$, and so on.

Among the four seismic stations used in this study, the receiver functions collected from 189 station have the best signal-to-noise ratio with fair azimuth coverage and obvious transform amplitude. Thus we take 189 station for example to describe the procedure used to retrieve the crust anisotropy. The azimuthal variation of the radial and transverse receiver functions are shown in Figure 4a. The receiver functions have clear amplitude variations at $0 \mathrm{~s}, 1 \mathrm{~s}, 2 \mathrm{~s}, 3 \mathrm{~s}$ and $5 \mathrm{~s}$, respectively, and the first phase at $0 \mathrm{~s}$ is correlated with the initial $\mathrm{P}$ wave. According to crustal
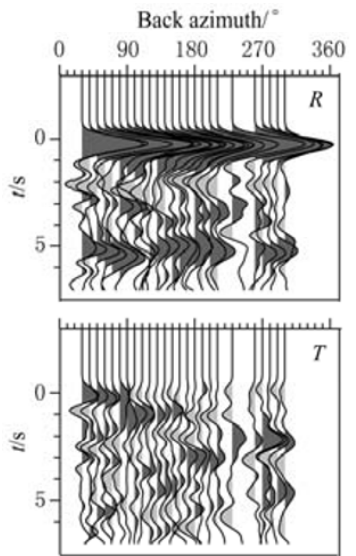

(a)
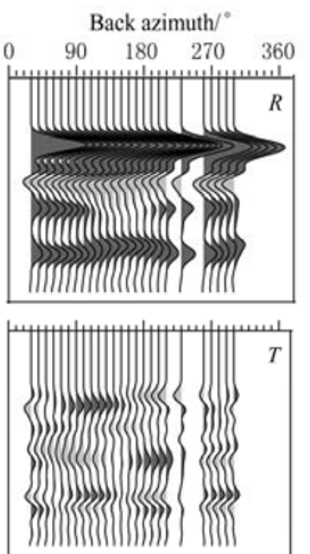

(b)

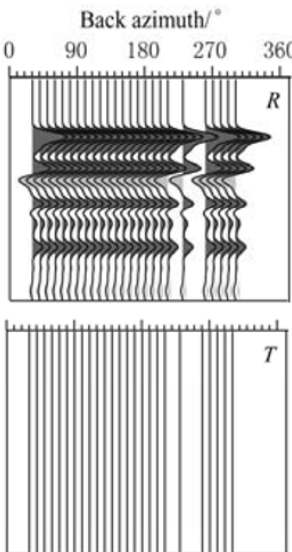

(c)

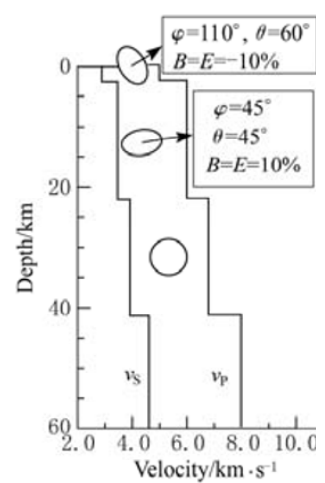

(d)

Figure 4 (a) Receiver functions obtained from records; (b) Synthetic receiver functions computed from the anisotropic model obtained by inversion; (c) Synthetic receiver functions computed from an isotropic model with the average velocity of the anisotropic model; (d) Crust velocity model from inversion. $R$ means radial component and $T$ transverse one 
model presented by ZHENG et al $(2006,2007)$ and JIA and ZHANG (2005), there is a shallow layer about $2 \mathrm{~km}$ thick at the surface in TMR. Synthetic testing shows that the phases at $1 \mathrm{~s}$ and $2 \mathrm{~s}$ are not P-S converted waves but the multiple wave phases. For the amplitude polarity at $3 \mathrm{~s}$ and $5 \mathrm{~s}$ are identical to the first arrival P-wave, we regard them as the P-S converted phases induced by velocity discontinuities at different depths, and the phase at $5 \mathrm{~s}$ is converted at the Moho discontinuity. In one word, a three-layered model can be a fair approximation to study the anisotropy beneath the seismic station.

The method of trial and error was used to retrieve the anisotropy parameters. A best fitted model was obtained as shown in Figure $4 \mathrm{~d}$ and the synthetic receiver functions were shown in Figure $4 \mathrm{~b}$. For fully understanding the relationship between anisotropy and receiver functions, we created an isotropy model based on the average velocity of the anisotropy model as shown in Figure $4 \mathrm{~d}$ and presented the synthetic receiver functions as shown in Figure 4c. After comparison of those figures, we can found that the anisotropy have noticeable influence on the receiver functions. The result, which is obtained based on waveform fitting using an isotropic model, would far from the real velocity model when existing significant anisotropy in the Earth.

\subsection{Inversion results}

We applied the same procedure, which is applied on 189 station, on the other stations, and finally obtained the crustal anisotropy parameters beneath the four seismic stations deployed at TMR in North China as shown in Table 1, as well as in Figure 5.
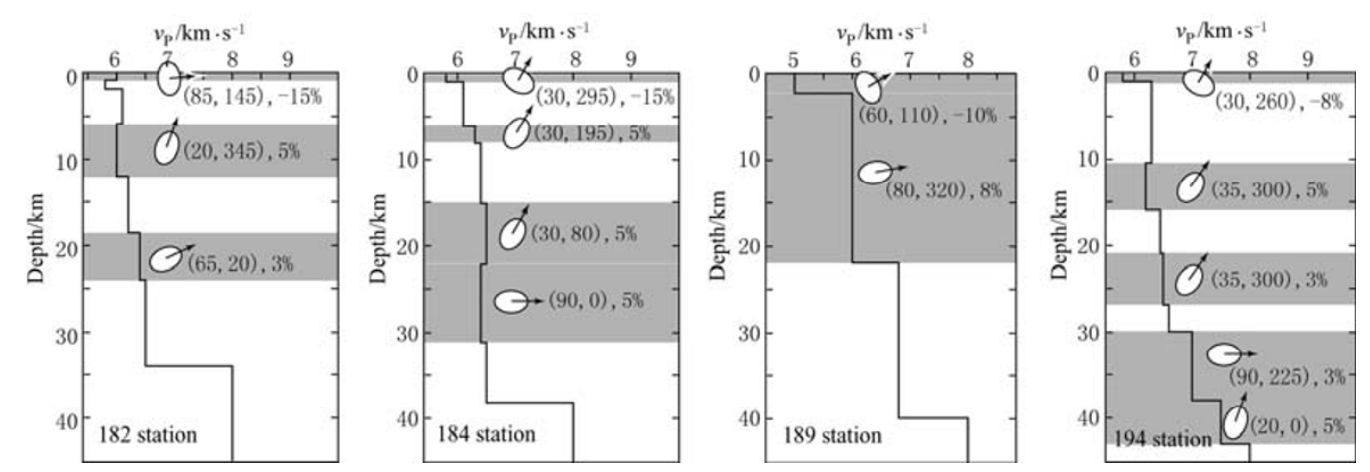

Figure 5 Illustration of the anisotropic velocity model

Gray areas show the layers with anisotropy and the numbers on them denote corresponding anisotropy parameters (plunge, trend) and magnitude

Figure 6 shows the receiver functions from records (left) and from synthetics (right) with azimuthal variation of the other three stations excepting 189 station. The upper and lower figures are corresponding to radial and transverse components respectively. We can see that, there is significant energy on the transverse component of receiver functions; in addition, patterns of receiver function amplitudes and arrival times on both radial and transverse components shows a systematic function of source-receiver back azimuth. The synthetic receiver functions computed for anisotropic model can fit the pattern and its azimuthal variation well. For some azimuth range, there are some differences between the synthetic and observed, especially on the transverse component. It may be due to the low signal-to-noise ratio of the transverse component or the rough model that we used in this study.

Our results indicated that there is significant anisotropy in both upper and lower crust. The surface layer at TMR in North China is approximately $1 \mathrm{~km}$ thick but has strong anisotropy. The 
anisotropy magnitude of about $8 \% \sim 15 \%$ was found in the upper crust with a slow axis and a smaller magnitude of about $3 \% \sim 5 \%$ in the lower crust with a fast axis.

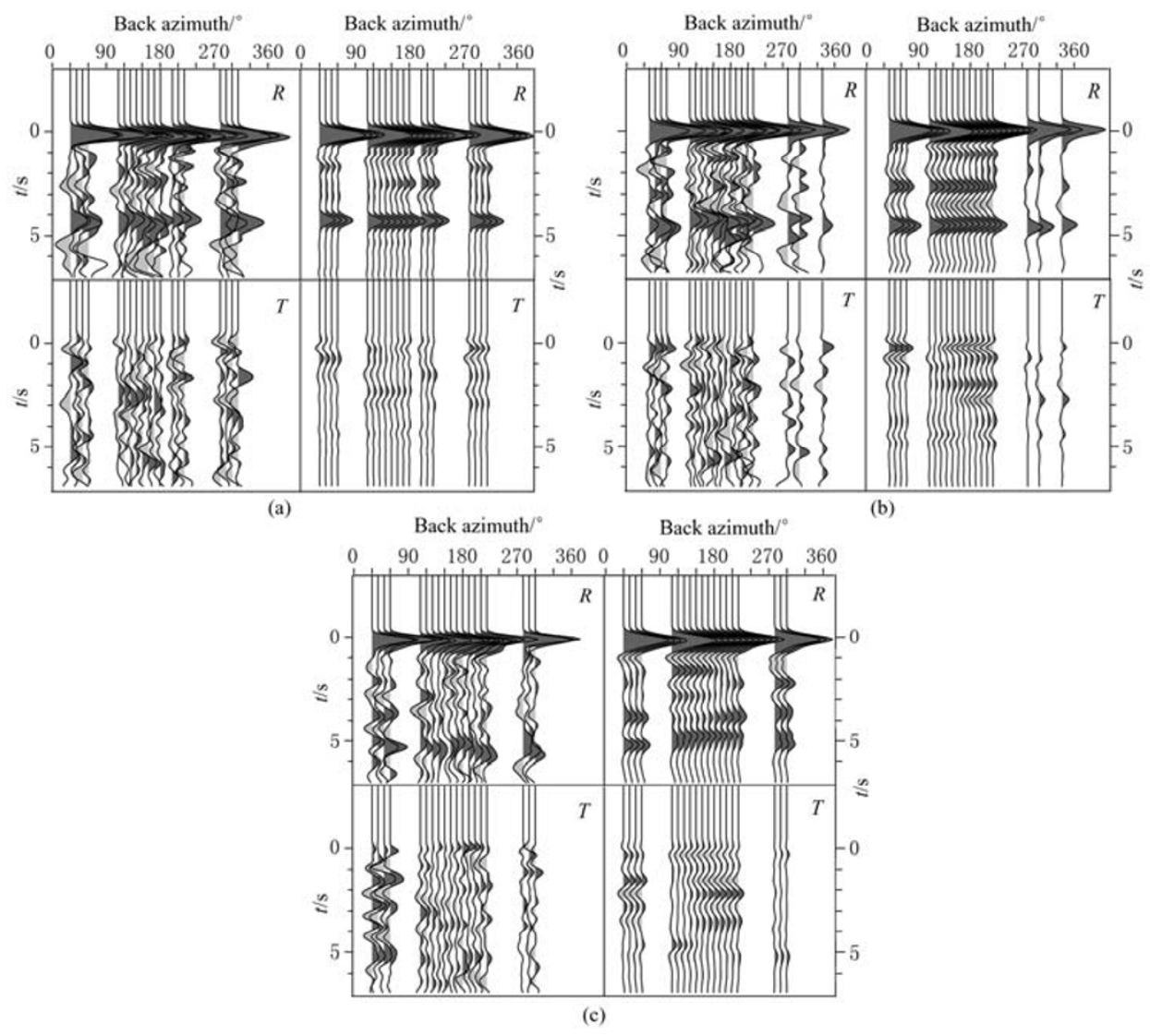

Figure 6 Receiver functions from record (left) and the synthetics computed from the velocity model from inversion (right)

(a) Station 182; (b) Station 184; (c) Station 194. $R$ means radial component and T transverse one

\section{Discussion and conclusions}

Seismic data have collected in this study from 20 linearly aligned seismic stations, trending NW-SE, numbered from $181\left(115.3^{\circ} \mathrm{E}, 39.0^{\circ} \mathrm{N}\right)$ to $200\left(113.5^{\circ} \mathrm{E}, 40.0^{\circ} \mathrm{N}\right)$. Through the analysis of the preliminary treatment of the seismic data, only the four selected stations show significant anisotropy with simple flat-layered structure and used to invert the anisotropic structure beneath the stations. The receiver functions of the most of unselected stations, excepting the few with too low signal-to-noise ratio to identify the seismic signals, also show azimuthal variations in various degrees. However, because of the strong noise and/or the presence of both anisotropy and lateral heterogeneity, it is hard to simulate the waveform of receiver functions with anisotropic models straightly.

The potential causes of crustal anisotropy are less straightforward. The sources of anisotropy may be one or both of the inherent anisotropy and induced anisotropy. The mineral grains with strong inherent anisotropy aligned along a predominant direction, due to the stress field and the 
flow in the asthenosphere or mantle, present significantly anisotropy in large scale, as well as the aligned microcracks, perhaps developed due to a nonhydrostatic stress field. The strata composed of many thin isotropic layers, named thin interbedded formations; in sedimentary rocks also present long-wavelength anisotropy. Moreover, the fluid, such as oil, gas and water in the microcracks, and the changes of sedimentary environments could also result in relatively uniform seismic anisotropy.

The crustal anisotropy has distinct characteristics in different area with both lateral and vertical variations because of the variety and complexity of anisotropy sources. Winterstein and Meadows (1991a, b) analyzed the data from Cymric, Railroad and Lost Hiccs Gap oil fields, and the results showed that orientation of symmetric axis and magnitude of anisotropy vary in different depths from surface to hundreds of meters downward. Lynn (1991) and Savage and Silver (1993) both got similar results by separately analyzing the local test records or teleseismic records. There is obvious inconsistency of the symmetry axes' orientations in different seismic stations, implying the different tectonic settings in a small local area, and in different depth, implying different formation mechanism and formation history of anisotropy. The TMR in North China has experienced many times of tectonic movements and the complexity of the crust structure may result in the difference in lateral and vertical. The inconsistency of our results deduced from the four stations also supports the above inference.

We can get much more information about the crustal anisotropic structure by analyzing the azimuthal variations of receiver functions, and the results we got here also can be used to complement and verify the results obtained by shear-wave splitting technique. The direction of fast axis and the delay time between the slow and fast shear wave can be obtained from teleseismic SKS wave splitting, and the source of anisotropy is generally regarded as coming from the mantle, while the contributions of the crustal anisotropy are ignored. However, the results from numerical simulation indicated that the crustal anisotropy may have strong effect on the mantle anisotropy parameters when using SKS wave splitting technique in some conditions, such as the directions of symmetry axis of the crust and mantle anisotropy are different from each other (Ruan et al, 2004). The presence of crustal anisotropy yield to the variety of the results obtained by various scholars or from different event record. Thus, we should consider both the contributions of crust and mantle anisotropy especially in North China where the magnitude of anisotropy is smaller than other areas.

The method of studying crustal anisotropy by analyzing the azimuthal variation of receiver functions can be applied to the area with complex anisotropic structure, and can get the anisotropy parameters including the magnitude, orientation and depth of anisotropy. Using this method we investigated the crustal anisotropic structure beneath the four stations aligned across the TMR in North China. Significant anisotropy with a slow symmetry axis in the upper crust and with a fast symmetry axis in the lower crust is found from our results, and may be associated with the local crustal fabrics in a small area. Thus the results from shear wave splitting should consider the effect of crustal anisotropy in different depth. Because of the absence of events in some azimuth, the low signal-to-noise ratio records and the tradeoff among the anisotropy parameters, the result inverted from the waveform of receiver functions occasionally exhibits a problem of solution non-uniqueness. Our results may be not consistent with the real crust model in details, but provide the definite seismic evidence of the presence of anisotropy within the crust at TMR in North China.

Acknowledgement We acknowledge the Broadband Seismic Array Laboratory, Institute of 
Geology and Geophysics, Chinese Academy of Science for providing the digital seismic data.

\section{References}

Anderson D L. 1961. Elastic wave propagation in layered anisotropic media [J]. J Geophys Res, 66: $2953-2963$.

Chen X. 1993. A systematic and efficient method of computing normal mode for multi-layered half-space [J]. Geophys $J$ Int, 115: 391-409.

Crampin S and Booth D C. 1985. Shear-wave polarization near the North Anatolian Fault: II. Interpretation in terms of crack-induced anisotropy [J]. Geophys J R Astron Soc, 83: 75-92.

DU Qi-zhen and YANG Hui-zhu. 2004. Viscoelastic wave equations of seismic multi-wave in fractured media [J]. Acta Physica Sinica, 53(8): 2 801-2 806 (in Chinese).

Hess H H. 1964. Seismic anisotropy of the uppermost mantle under ocean [J]. Nature, 203: 629-631.

Iidaka T and Niu F L. 2001. Mantle and crust anisotropy in the eastern China region inferred from waveform splitting of SKS and PpSms [J]. Earth Planets Space, 53: 159-168

JIA Shi-xu and ZHANG Xian-kang. 2005. Crustal structure and comparison of different tectonic blocks in North China [J]. Chinese $J$ Geophys, 48(3): 611-620 (in Chinese).

Karato S I. 1998. Seismic anisotropy in the deep mantle, boundary layers and the geometry of mantle convection [J]. Pure Appl Geophys, 151: 565-587.

Levin V and Park J. 1997. Crustal anisotropy in the Ural Mountains foredeep from teleseismic receiver functions [J]. Geophys Res Lett, 24: $1283-1286$.

Li J, Tian B F, Wang W M, et al. 2007. Lateral variation in the sedimentary structure of west Bohai Bay Basin inferred from P-multiple receiver functions [J]. Bull Seism Soc Amer, 97(4): 1355-1363.

Liu K H, Gao S S, Gao Y, et al. 2008. Shear wave splitting and mantle flow associated with the deflected Pacific slab beneath northeast Asia [J]. J Geophys Res, 113: B01305.

LIU Qi-yuan, Kind R, LI Shun-cheng. 1997. Receiver function at the stations of the Chinese digital seismic network (CDSN) and their nonlinear inversion [J]. Chinese J Geophys, 40(3): 356-368 (in Chinese).

LIU Xi-qiang, ZHOU Hui-lan, LI Hong, et al. 2001. Anisotropy of the upper mantle in Chinese mainland and its vicinity [J]. Acta Seismologica Sinica, 14(4): 359-370.

Lu S N, Zhao G C, Wang H C, et al. 2008. Precambrian metamorphic basement and sedimentary cover of the North China Craton: Review [J]. Precambrian Research, 160: 77-93.

LUO Yan, HUANG Zhong-xian, PENG Yan-ju, et al. 2004. A study on SKS wave splitting beneath the China mainland and adjacent regions [J]. Chinese J Geophys, 47(5): 812-821 (in Chinese).

Lynn H B. 1991. Field measurements of azimuthal anisotropy: First 60 meters, San Francisco Bay area, CA, and estimation of the horizontal stresses' ratio from Vs1/Vs2 [J]. Geophysics, 56(6): 822-832.

Montagner J P. 1998. Where can seismic anisotropy be detected in the earth's mantle? In boundary layers [J]. Pure Appl Geophys, 151: 223-256.

Owens T J, Zandt G, Taylor S R. 1984. Seismic evidence for an ancient rift beneath the cumber- land Plateau, Tennessee: A detailed analysis of broadband teleseimic P waveforms [J]. J Geophys Res, 89: 7 783-7 795.

Ozacar A A and Zandt G. 2004. Crustal seismic anisotropy in central Tibet: Implications for deformational style and flow in the crust [J]. Geophys Res Lett, 31: L23601.

Park J. 1996. Surface waves in layered anisotropic structures [J]. Geophys J Int, 126: 173-184.

RUAN Ai-guo, WANG Chun-yong, LI Qing-he, et al. 2004. Effect of the crust on the analysis of the upper mantle anisotropy [J]. Chinese J Geophys, 47(3): 441-448 (in Chinese).

Savage M K and Silver P G. 1993. Mantle deformation and tectonics: Constraints from seismic anisotropy in the western United States [J]. Phys Earth Planet Inter, 78: 207-227.

Savage M K. 1998. Lower crustal anisotropy or dipping boundaries: Effects on receiver functions and a case study in New Zealand [J]. $J$ Geophys Res, 103(B7): 15 069-15087.

Sherrington H F, Zandt G, Frederiksen A. 2004. Crustal fabric in the Tibetan Plateau based on waveform inversions for seismic anisotropy parameters [J]. J Geophys Res, 109: B02312.

TIAN Bao-feng, LI Juan, WANG Wei-min, et al. 2008. Crust anisotropy of Taihangshan Mountain Range in North China inferred from receiver functions [J]. Chinese J Geophys, 51(5): in press (in Chinese).

Tommasi A, Tikoff B, Vauchez A. 1999. Upper mantle tectonics: Three-dimensional deformation, olivine crystallographic fabrics and seismic properties [J]. Earth Planet Sci Lett, 168: 173-186.

Tsvankin I and Thomsen L. 1994. Nonhyperbolic reflection moveout in anisotropic media [J]. Geophysics, 59: $1290-1304$.

Winterstein D F and Meadows M A. 1991b. Changes in shear-wave polarization azimuth with depth in Cymric and Railroad Gap oil fields [J]. Geophysics, 56(9): 1349-1 364.

Winterstein, D F and Meadows M A. 1991a. Shear-wave polarization and subsurface stress directions at Lost Hills field [J]. Geophysics, 56(9): 1331-1348.

WU Jing, GAO Yuan, CHEN Yun-tai, et al. 2007. Seismic anisotropy in the crust in northwestern capital area of China [J]. Chinese $J$ Geophys, 50(1): 209-220 (in Chinese).

Wu Q J, Li Y H, Zhang R Q, et al. 2007a. Wavelet modelling of broad-band receiver functions [J]. Geophys J Int, 170: $534-544$.

Wu Q J, Li Y H, Zhang R Q, et al. 2007b. Receiver functions from autoregressive deconvolution [J]. Pure Appl Geophys, 164: 2 175-2 192. 
WU Qing-ju, TIAN Xiao-bo, ZHANG Nai-ling, et al. 2003. Receiver function estimated by maximum entropy deconvolution [J]. Acta Seismologica Sinica, 16(4): 404-412.

Zhao L and Zheng T Y. 2005. Using shear wave splitting measurements to investigate the upper mantle anisotropy beneath the North China Craton: Distinct variation from east to west [J]. Geophys Res Lett, 32: L10309.

ZHENG Hai-shan and ZHANG Zhong-jie. 2005. Synthetic seismograms of nonlinear seismic waves in anisotropic (VTI) media [J]. Chinese J Geophys, 48(3): 660-671 (in Chinese).

ZHENG Si-hua and GAO Yuan. 1994. Azimuth anisotropy of lithosphere of continental China [J]. Acta Seismologica Sinica, 7(2): $177-186$.

Zheng T Y, Chen L, Zhao L, et al. 2006. Crust-mantle structure difference across the gravity gradient zone in North China Craton: Seismic image of the thinned continental crust [J]. Phys Earth Planet Inter, 159: 43-58.

Zheng T Y, Chen L, Zhao L, et al. 2007. Crustal structure across the Yanshan belt at the northern margin of the North China Craton [J]. Phys Earth Planet Inter, 161: 36-49. 